\title{
Calibrating damping rates with LEGACY` linewidths
}

\author{
Günter Houdek ${ }^{1, \star \star}$ \\ ${ }^{1}$ Stellar Astrophysics Centre, Aarhus University, DK-8000 Aarhus C, Denmark
}

\begin{abstract}
Linear damping rates of radial oscillation modes in selected Kepler stars are estimated with the help of a nonadiabatic stability analysis. The convective fluxes are obtained from a nonlocal, time-dependent convection model. The mixing-length parameter is calibrated to the surface-convection-zone depth of a stellar model obtained from fitting adiabatic frequencies to the LEGACY ${ }^{\star}$ observations, and two of the three nonlocal convection parameters are calibrated to the corresponding LEGACY ${ }^{\star}$ linewidth measurements. The atmospheric structure in the 1D stability analysis adopts a temperature-optical-depth relation derived from 3D hydrodynamical simulations. Results from 3D simulations are also used to calibrate the turbulent pressure and to guide the functional form of the depth-dependence of the anisotropy of the turbulent velocity field in the 1D stability computations.
\end{abstract}

\section{Introduction}

We use LEGACY [14] linewidths and frequencies of selected solar-type Kepler stars, together with 3D hydrodynamical simulation results $[15,16]$, for calibrating the global stellar and nonlocal convection parameters in 1D stability computations. Additionally to exploiting the seismic diagnostic of observed oscillation frequencies, linewidth measurements provide further diagnostic information about the physical processes prevailing in the outer superadiabatic boundary layers where convective transport becomes inefficient. It is in these outer stellar layers where solar-like oscillations are excited stochastically to the observed oscillation amplitudes and damped by various processes including the interaction between oscillations and convection. Therefore, a time-dependent convection model is required for describing these physical processes, including the pulsationally perturbations to both the convective heat (enthalpy) and momentum (turbulent pressure) fluxes. A consistent inclusion of turbulent pressure in the equilibrium structure is, however, only possible within the framework of a nonlocal formulation of convection [7]. Such a convection model is adopted here $[7,8]$. Within the generally assumed approximations for constructing a 1D convection model, such as the Boussinesq approximation [17] (for a recent review see [10]), the turbulent fluxes are consistently estimated in both the equilibrium and nonadiabatic pulsation calculations. A nonlocal convection formulation typically has additional (nonlocal) parameters which need calibration. Here we use results from 3D hydrodynamical simulations and linewidth measurements from Kepler data for calibrating these additional (three) nonlocal convection parameters. We also

\footnotetext{
${ }^{\star}$ data provided by Mikkel N. Lund (e-mail: mikkelnl@phys.au.dk)

$\star \star$ e-mail: hg@phys.au.dk
}

include an analytical description for the variation of the anisotropy of the turbulent velocity field, the functional form of which being guided by 3D simulations [15].

\section{Model computations}

The 1D nonlocal model calculations are carried out essentially in the manner described by [9] and [2] but include, in addition, a description for the variation of the turbulent velocity anisotropy with stellar depth, and a temperatureoptical-depth $(T-\tau)$ relation derived from 3D simulations [16]. The convective heat flux and turbulent pressure are obtained from a nonlocal generalization of the mixinglength formulation $[7,8]$. In this generalization three more (nonlocal) parameters, $a, b$ and $c$, are introduced which control the spatial coherence of the ensemble of eddies contributing to the total convective heat flux $(a)$ and turbulent pressure $(c)$, and the degree to which the turbulent fluxes are coupled to the local stratification $(b)$. The effects of varying these nonlocal parameters on the solar structure and oscillation properties were discussed in detail by [1].

The nonlocal parameter $c$ is calibrated such as to have the maximum value of the turbulent pressure, $\max \left(p_{\mathrm{t}}\right)$, in the 1D nonlocal model to agree with the 3D simulation result by [15]. The depth-dependence of the anisotropy $\Phi:=\overline{\boldsymbol{u} \cdot \boldsymbol{u}} / \overline{w^{2}}$ of the convective velocity field $\boldsymbol{u}=(u, v, w)$ (an overbar denotes an ensemble average) is described by an analytical function, guided by $3 \mathrm{D}$ simulation results [15]. The remaining nonlocal parameters $a$ and $b$ cannot be easily obtained from the 3D simulations and are therefore calibrated such as to have a good agreement between calculated damping rates and LEGACY linewidths over the whole measured frequency range. The mixing length is calibrated such as to obtain the surface-convection depth of the frequency-calibrated evolutionary model calculated 

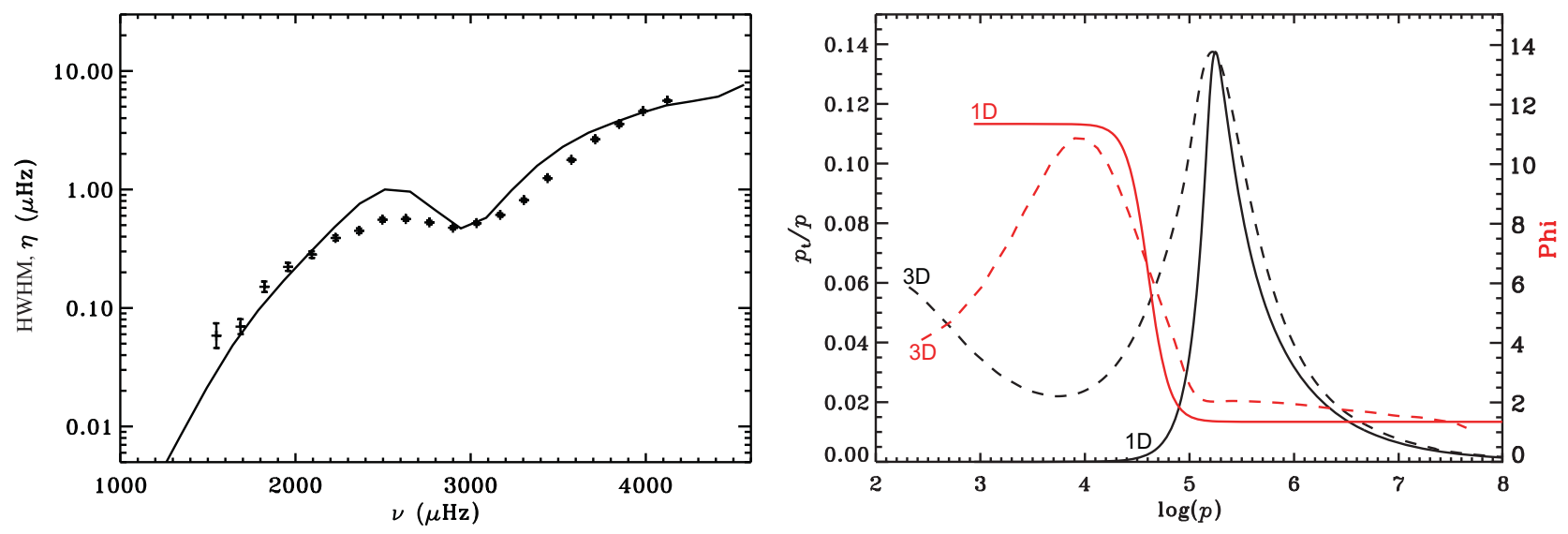

Figure 1: Comparison of solar stability computations with BiSON data and results from 3D hydrodynamical simulations. The left panel shows theoretical damping rates $\eta$, in units of cyclic frequency (solid curve), as functions of cyclic oscillation frequency $v$, together with half-width-at-half-maximum (HWHM) measurements (symbols with error bars) of the spectral peaks in an acoustic power spectrum obtained by BiSON [2]. The right panel compares profiles of the turbulent pressure $p_{\mathrm{t}}$ over the total pressure $p$ (black curves) and turbulent-velocity anisotropy $\Phi$ (red curves) between a 3D simulation (dashed curves) and a calibrated 1D solar model (solid curves).
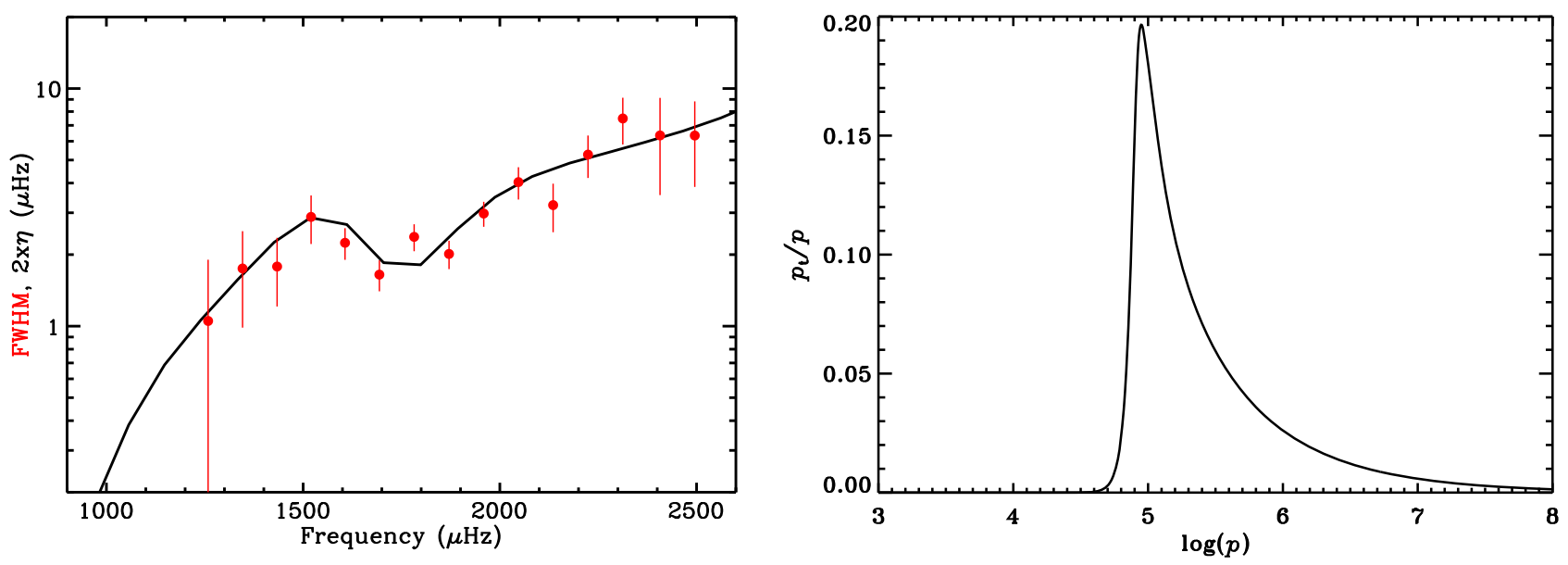

Figure 2: Results of a stability calculation for the Kepler star KIC12009504 (Dushera) with an effective temperature $T_{\text {eff }}=6217 \mathrm{~K}$ and surface gravity $\log g=4.214$. The left panel compares LEGACY linewidths [14] (red symbols with $1 \sigma$ error bars) with twice the theoretical damping-rate estimates, $2 \times \eta$ (solid curve), in units of cyclic frequency, as functions of cyclic oscillation frequency. The right panel shows the profile of (3D-calibrated) turbulent pressure $p_{\mathrm{t}}$ over the total pressure $p$ in the 1D stellar model.

with ASTEC [4]. The ASTEC evolutionary model is obtained by minimizing the differences between observed (LEGACY) and adiabatically computed (ADIPLS [5]) oscillation frequencies, including corrections for surface effects according to [6].

Both the nonlocal envelope and pulsation calculations of the stability analyses assume the generalized Eddington approximation to radiative transfer [18]. The temperature gradient in the plane-parallel atmosphere is corrected by using a radially varying Eddington factor fitted to $T-\tau$ relations obtained from $3 \mathrm{D}$ simulation results [16]. The abundances by mass of hydrogen and heavy elements are adopted from the frequency-calibrated evolutionary calculations. The opacities are obtained from the OPAL tables [12], supplemented at low temperature by tables from [13].
The equation of state includes a detailed treatment of the ionization of $\mathrm{C}, \mathrm{N}$, and $\mathrm{O}$, and a treatment of the first ionization of the next seven most abundant elements [3]. The integration of stellar-structure equations starts at an optical depth of $\tau=10^{-4}$ and ends at a radius fraction $r / \mathrm{R}_{\odot}=0.2$.

The linear nonadiabatic pulsation calculations are carried out using the same nonlocal convection formulation with the assumption that all eddies in the cascade respond to the pulsation in phase with the dominant large eddies. A simple thermal outer boundary condition is adopted at the temperature minimum where for the mechanical boundary condition the solutions are matched smoothly onto those of a plane-parallel isothermal atmosphere. At the base of the model envelope the conditions of adiabaticity and vanish- 

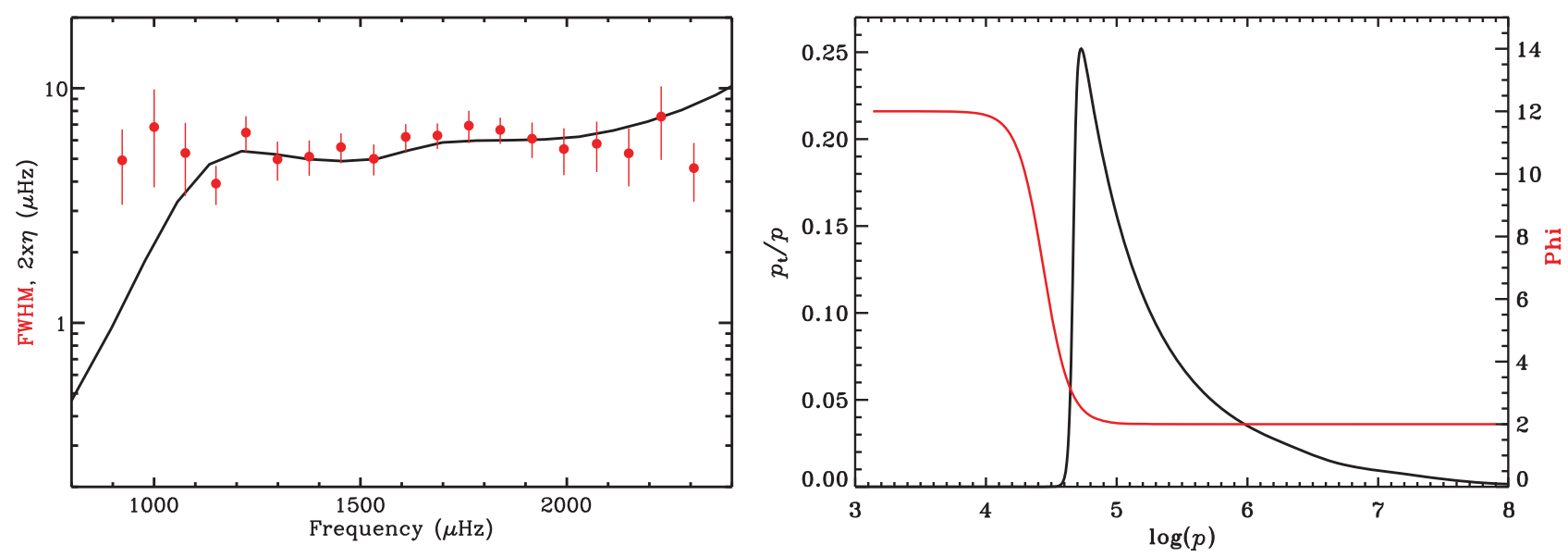

Figure 3: Results of a stability calculation for the Kepler star KIC11253226 (Tinky) with an effective temperature $T_{\text {eff }}=6715 \mathrm{~K}$ and surface gravity $\log g=4.171$. The left panel compares LEGACY linewidths [14] (full-width-at-half-maximum FWHM, red symbols with $1 \sigma$ error bars) with twice the theoretical damping-rate estimates, $2 \times \eta$ (solid curve), in units of cyclic frequency, as functions of cyclic oscillation frequency. The right panel shows the profiles of (3D-calibrated) turbulent pressure $p_{\mathrm{t}}$ over the total pressure $p$ (black curve) and turbulent-velocity anisotropy $\Phi$ (red curve) in the 1D stellar model.

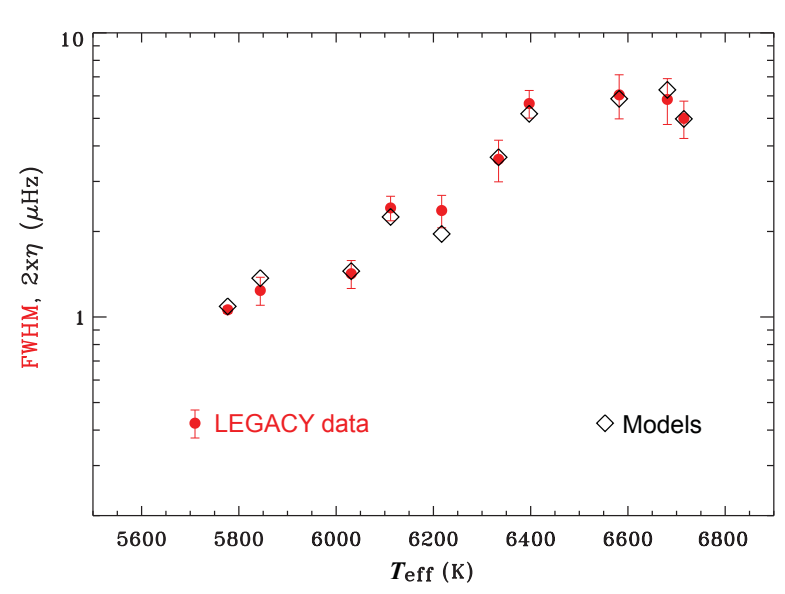

Figure 4: Comparison of measured LEGACY linewidths (fullwidth-at-half-maximum FWHM, red, filled circles with $1 \sigma$ error bars, [14]) with twice the estimated damping rates (black, open diamond symbols), in units of cyclic frequency, for 9 Kepler stars and the Sun (for the Sun BiSON linewidths [2] are shown). Results are plotted as functions of the model's effective temperatures and at the frequency $v \simeq v_{\max } \propto g T_{\mathrm{eff}}^{-1 / 2}$, assuming a solar $v_{\max } \simeq 3100 \mu \mathrm{Hz}$.

ing of the displacement eigenfunction are imposed. Only radial $\mathrm{p}$ modes are considered.

\section{Results}

We first tested the stability analysis against solar data provided by the BiSON group [2] (see also [11]). The results are illustrated in Figure 1. The left panel compares radial damping-rate estimates $\eta$, in units of cyclic frequency, with half-width-at-half-maximum (HWHM) measurements of the spectral peaks in the acoustic power spectrum. The power spectrum was obtained from a BiSON time-series collected over an epoch of 3456 days [2]. The right panel compares the profiles of turbulent pressure $p_{\mathrm{t}}$ and convective velocity anisotropy $\Phi$ as functions of the logarithm of the total pressure $p$ in the outer stellar layers between a 3D solar simulation [15] and a calibrated $1 \mathrm{D}$ model. Only the maximum value of $p_{\mathrm{t}}:=\overline{\rho w w}(\rho$ is density) in the 1D model was calibrated to the 3D simulation results by adjusting the nonlocal convection parameter $c$. The agreement between the model and simulation is particularly satisfactorily in the deeper stellar layers where the modes are propagating. Note that in the outer layers with $\log p \lesssim 5.3$ the modes are evanescent (e.g. [11]). The increasing difference in $p_{\mathrm{t}}$ between simulation (dashed curve) and model (solid curve) with decreasing total pressure $p$ is predominantly a consequence of neglecting in a Boussinesq fluid the acoustic flux (H.-G. Ludwig, personal communication), generated by the convective fluctuations. These differences in stellar stratification have, however, little effect on the acoustic oscillation properties, for these differences are confined in the evanescent layers (see also [11]).

Next we estimated damping rates in 9 solar-type Kepler stars with effective temperatures $5844 \mathrm{~K} \leq T_{\text {eff }} \leq$ $6715 \mathrm{~K}$. Results for the frequency-dependence of the estimated damping rates and profiles of turbulent pressure of the corresponding 1D stellar stratifications are illustrated in Figures 2 and 3 for two models with different effective temperatures. As in the solar case, $\max \left(p_{\mathrm{t}}\right)$ in the $1 \mathrm{D}$ model was calibrated such as to match $\max \left(p_{\mathrm{t}}\right)$ in the $3 \mathrm{D}$ simulations [15].

Figure 4 compares LEGACY linewidths [14] (red, filled circles with error bars) with estimated damping rates (black, open diamonds) for 9 selected Kepler stars and for the Sun. Results are plotted at the frequency $v_{\max }$ of maximum oscillation amplitude, determined from the scaling relation $v_{\max } \propto g T_{\mathrm{eff}}^{-1 / 2}(g$ being surface gravity) and assuming a solar $\nu_{\max } \simeq 3100 \mu \mathrm{Hz}$. The agreement between 
observations and models is very satisfactorily. Moreover, all 1D models have global stellar parameters determined from minimizing the differences between observed and adiabatically computed oscillation frequencies [14], calibrated $\max \left(p_{\mathrm{t}}\right)$ values, depth-dependent functional forms of the turbulent-velocity anisotropy $\Phi$ and $T-\tau$ relations as suggested by $3 \mathrm{D}$ simulations $[15,16]$.

\section{Acknowledgements}

We thank Mikkel N. Lund for providing the LEGACY linewidths and Jørgen Christensen-Dalsgaard for the global stellar parameters of the frequency-calibrated ASTEC evolutionary models. Funding for the Stellar Astrophysics Centre is provided by The Danish National Research Foundation (Grant DNRF106).

\section{References}

[1] Balmforth N. J., MNRAS 255, 603 (1992)

[2] Chaplin W. J., Houdek G., Elsworth Y., Gough D. O., Isaak G. R., New R., MNRAS 360, 859 (2005)

[3] Christensen-Dalsgaard J., MNRAS 199, 735 (1982)

[4] Christensen-Dalsgaard J., Ap\&SS 316, 13 (2008)

[5] Christensen-Dalsgaard J., Ap\&SS 316, 113 (2008)
[6] Christensen-Dalsgaard J., ESF Conference: "The Modern Era of Helio- and Asteroseismology” (M. Roth ed., Obergurgel), Astron. Nachr. 333, 914 (2012)

[7] Gough D. O., Lecture Notes in Physics 71, (Spiegel E. A., Zahn J.-P., eds, Springer, New York), 15 (1977a)

[8] Gough D. O., ApJ 214, 196 (1977b)

[9] Houdek G., Balmforth N. J., Christensen-Dalsgaard J., Gough D. O., AA 351, 582 (1999)

[10] Houdek G., Dupret M.-A., Living Rev. Sol. Phys., 12:8 (2015)

[11] Houdek G., Trampedach R., Aarslev M. J., Christensen-Dalsgaard J., MNRAS 464, 124 (2017)

[12] Iglesias C. A., Rogers F. J., ApJ 464, 943 (1996)

[13] Kurucz R. L., NATO Series C 341, (Crivellari L., Hubeny I., Hummer D. G., eds, D. Reidel Publishing Co., Trieste Italy), 441 (1992)

[14] Lund M. N., Silva Aguirre V., Davies G. R., Chaplin W. J., Christensen-Dalsgaard J. et al., submitted to ApJ (2016)

[15] Trampedach R., Asplund M., Collet R., Nordlund Å., Stein R. F., ApJ 769, 18 (2013)

[16] Trampedach R., Stein R. F., Christensen-Dalsgaard J., Nordlund Å., Asplund M., MNRAS 442, 805 (2014)

[17] Spiegel E. A., Veronis G., ApJ 131, 442 (1960)

[18] Unno W., Spiegel E. A., PASJ 18, 85 (1966) 